\title{
Discussion on Multi-sensor Detector Fusion of Internet of Things in Vehicle Management
}

\author{
Wei He, ${ }^{*} \mathrm{Qi} \mathrm{Li}$, Wei hua Sun \\ The third research institute of the ministry of public security of P.R.C \\ Ilheweii@126.com, fw339lq@126.com
}

\begin{abstract}
Keywords: Multi-sensor, Detector Fusion, IOT
Abstract. In this paper, multi-sensor technology fusion of internet of things, such as radio frequency Identification(RFID), Beidou navigation satellite system(BDS ), mobile communication, video recognition applied in vehicle management is discussed. In this study, fabricating multi-sensor detector fusion applied in toll collection of highway system and automatic supervising fake vehicle license plate system. It can effectively improve the efficiency of toll collection of highway and supervise fake vehicle license, violation vehicle, and provide accurate management solution for vehicle management agency.
\end{abstract}

\section{Introduction}

As rapid development of economy and vehicle population, vehicle management problems, such as toll collection, fake vehicle license, violation vehicle and traffic accidents etc. have caused more and more time waste, property loss and social security. To resolve these problems, intelligent transportation system (ITS) is being developed in many countries with an aim to improve road traffic efficiency and safety $[1,2]$. The major parameters for traffic monitoring systems are the speed and direction of the vehicle on the road. A conventional way to obtain this information is using solo cameras which need human labor and expensive cost. Another way is to use inductive loops beneath the road, which needs an expensive installation and maintenance cost. Besides, the conventional ITS with solo video capture has many information blind spot which cannot identify a cloning vehicle license or other traffic accident. At the same time, the accuracy of video capture is also a problem due to its sensitive to weather. Multi-sensor detectors fusion is the method of dealing with information from single or multiple sensors (or sources), which is used for automatic detection, connection, correlation, estimation and combination in multi-level and multi-facet, thus to obtain the more accurate description and identity estimattion such as target parameters, features, events and behaviors [3]. Sensor is an important tool to obtain information, and its role is similar to human sense organs. Multi-sensor detector fusion technology will be a trend in future[4-7] which can obtain more reliable, more complete convergence information by integrating data from a variety of similar structure or heterogeneous sensors compared with single sensor. Multi-sensor detector fusion technology of internet of things breaks through the expression limitation of single sensor and separation, avoids information blind spot of single sensor and enhances the result quality of processing multiple sources information, which is conducive to make a judgment and a decision during the target recognition. In this paper, we apply multi-sensor detector technology fusion based on internet of things in real-time toll collection of highway and automatic supervising fake vehicle license plate with the aim to provide high efficient management solution for vehicle.

\section{Real-time toll collection in highway}

Now, the highway has become more and more important in modern road network and the traditional manual toll collection has clearly exposed its weakness. Non-stop automatic toll collection is now imperative in highway systems. With the advent of the ITS, the establishment of electronic toll collection system (ETC) was been researching by the world used in the highway, bridge and tunnel[8-10]. Most ETC was based on RFID technology[9-10]. Throwing information change between electronic labels that held vehicle and RFID antennas that was fixed the roadside or door 
frame, the center control computer can identify the road user by the information stored in the electronic label and deduct the pike from the road user advance stored card or bank account. Its obvious advantage is toll collection without parking. Thereby it can significantly improve the dispose efficiency of the toll station and the traffic ability of toll road. But, the current management model, for example in China, is mostly like this: when the vehicles enter the highway, get the charge card in the toll stations which records the time, signs of the toll station, type of vehicle fees. Contrast to traditional manual toll collection, ETC has provided some solutions to the traffic jam, delay and some other problems, can radically enhance the safety and efficiency of road operation and achieve safe road traffic environment. But there are still much shortcoming of ETC, for example, it is a short distance(normally less than 10m) communication technology which don't support real-time communication of all road. Furthermore, it needs many separated toll stations scattered at entrance and exit to support system.

Now, the emergence of internet of things breaks the conventional thinking[11]. Past ideas separate the physical infrastructure and IT infrastructure. In the time of internet of things, roads, cars will be integrated unified infrastructure with the sensors, net. All items will be connected to the sensors such as RFID devices, camera, global positioning systems, etc. In this paper, the automatic toll collection of highway system is designed. When the vehicles enter the toll station, the drivers need to apply for an initialized smart card (or radio frequency card) which stores the simple information of the car such as license plate number from the toll station. As is shown in Figure 1, this smart card has RFID chip, cellphone chip and BDS chip. In this system, RFID tag works at $2.4 \mathrm{G}$ band. This RFID system have characteristics of far distance communication about $2000 \mathrm{~m}$. The RFID chip having unique ID can be used as electronic wallet which is integrated with cellphone chip and BDS chip. The cellphone chip and BDS chip can communication with control center and also have route navigation function. The fee of drivers can charged automatically in electronic wallet at toll collection website of highway system or set his electronic wallet related with credit card of bank. The RFID chip have high safety encryption to support the fee paying. When the car driving on highway, as shown in Figure 2, the smart card could send the driving information such as the license plate number, the driving route and monitoring station that the vehicle passed etc to the monitor base station and control center by the satellite communication or mobile communication facilities at any time. All toll can be charged by website of toll collection system. In this real-time toll collection system, toll can be precisely charged at different route although at the same entrance and the exit. As is shown in Figure 3, there are three routes between A entrance and B exit, they are the routes of $\mathrm{ACB}, \mathrm{ADB}$ and $\mathrm{AFB}$. each route has its toll price. when one vehicle passes through the same entrance and the same exit, but along different route, it may be collected with different toll automatically according to the route navigated by BDS or mobile communication.

To monitoring precisely, there should be monitoring station at entrance and exit. The monitoring station, which integrated RFID, camera and speed sensors etc, can detect detail information, such as electronic license plate number from RFID system, passing speed, physical license plate number, type and color of vehicle by intelligent video recognition and speeding sensor.

This designed real-time toll collection system attempts to use multi-sensor detector technology fusion of internet of things, establishing a concept model of highway vehicle information detection based on the technology of internet of things. In this design, the toll collection can been done automatically when the vehicle pass through toll collection station at free flow speed without stopping at any toll collection station. In this system, the accurate statistical models of traffic flow, forecast traffic flow in a short term still need to be united with traditional statistical methods of traffic flow. This designing analyzed the networking toll collection management system, which will provide a reference to non-stop toll collection system of the highway in the future. 


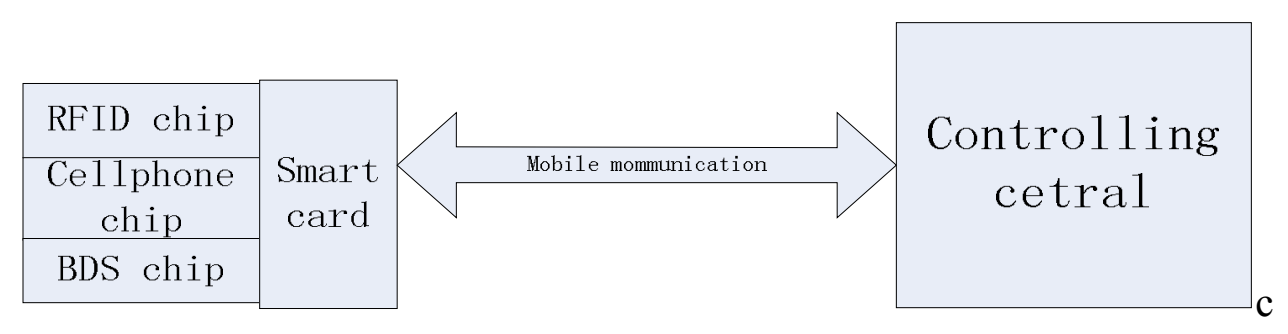

Figure 1 The schematic of the smart card and its communication

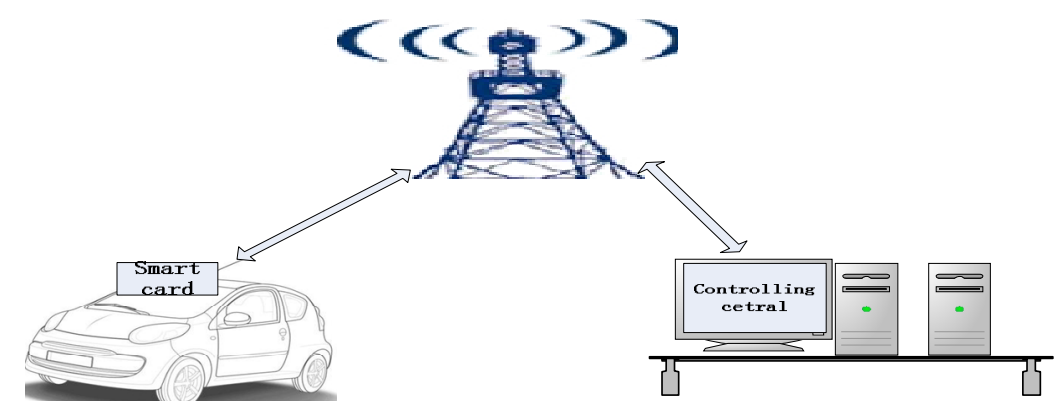

Figure 2 The schematic of multi-sensor detector technology fusion based on internet of things

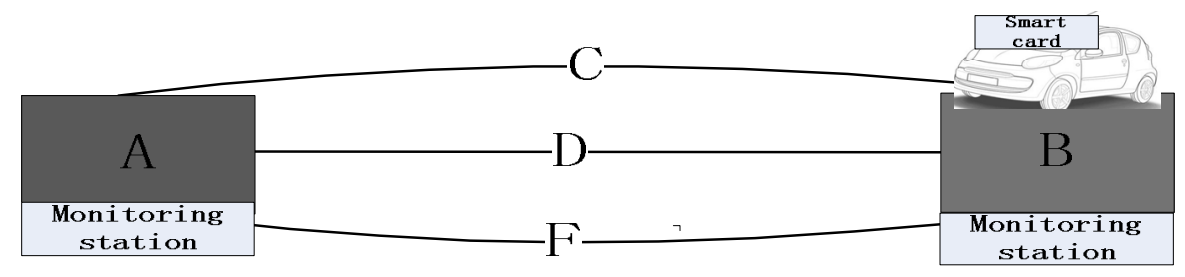

Figure 3 the Diagram of different route between same point of entrance and exit

\section{Automatically superviseing fake vehicle license plate}

At present, more and more vehicles run business for profit, such as taxis and trucks etc. becuase the owner of these business vehicle need pay high fee to applying for license authorized and approved by the government administrator. So, to avoid high fee, some many owner run vehicles with fake vehicle license plate on road. To resolve these problems, this study fabricates an automatically supervising fake vehicle license plate system using multi-sensor detector fusion technology of internet of things to provide accurate management solution for vehicle management agency.

Figure4 is the workflow chart of system of multi-sensor detector technology fusion. When a vehicle with RFID tag passes through one road, the RFID, video recognition works simultaneously. In this frame model, all multi-sensor information is converged by total control chassis of IPC( industrial personal computer). The multi- information include the electronic license plate number from RFID system, the physical license plate number, type and color of vehicle by Intelligent video recognition, and the speeding of vehicle, etc. By automatically detecting, logical judgment, estimating and combining from multi-level and multi-facet in the control chassis of IPC, the accurate information of vehicle, such as license plate number, type and color of vehicle, speeding, running time, place and so on is obtained. Then the control chassis of IPC can transmit all information of vehicle to control centre by internet. 


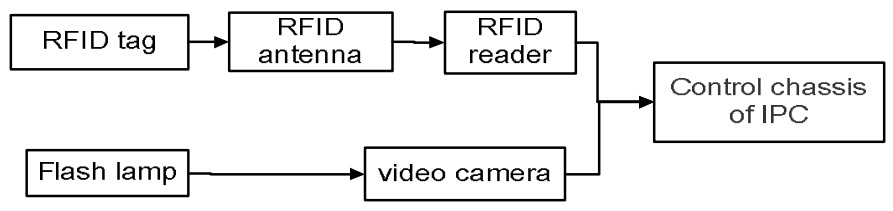

Figure4 The workflow chart of system of multi-sensor detector technology fusion

\section{Conclusion}

In this paper, real-time toll collection of highway system and automatic supervising fake vehicle license plate system using multi-sensor technology fusion of internet of things is presented. In the system of real-time toll collection of highway, the terminal smart card of vehicle with multi-sensor and its function of real-time toll collection is stressed. In the system of automatic supervising fake vehicle license plate, the supervising base station with multi-sensor and its supervising illegal vehicles is focused on. In both solutions, there are much same characteristics of multi-sensor of internet of things, real-time, free flow speed of vehicle and so on. In all, the result of this study can be proposed to complete efficiently reference instead of single sensor for vehicle management agency.

\section{References}

1.T. Dao, K. Leung, C.Clark, Jan. Huissoon. Co-operative Lanelevel Positioning Using Markov Localization. IEEE Transactions on Intelligent Transportation Systems, vol.9, no.1, 2008, pp. 48-57.

2. Daiheng Ni. Determining Traffic-flow Characteristics by Definition for Application in ITS. Intelligent Transportation Systems, IEEE Transactions on, Volume: 8, 2006, pp: 181-187.

3. Hengqing Tong, Xujie Zhao and Chao Yu. Multi-sensor Intelligent Transportation Monitoring System Based on Information Fusion Technology. 2007 International Conference on Convergence Information Technology, pp1726-1732.

4 . Zhou Fang, Han Liyan. A Survey of Multi-sensor Information Fusion Technology. Journal of Telemetry, Tracking and Command, 2006, 27(3), pp1-7.

5. Tsu-Tian Lee, Hsin-Han Chiang, Jau-Woei Perng, etc.. Multi-sensor Information Integration on DSP Platform for Vehicle Navigation Safety and Driving Aid. Proceedings of the 2009 IEEE International Conference on Networking, Sensing and Control, Okayama, Japan, March 26-29, 2009, pp653-658.

6. Liu Jiang, Wang Yun-peng. Multi-sensor based Vehicle Autonomous Navigation for Vehicle Infrastructure Integration: Concept and Simulation Analysis. 2011 International Conference on Transportation, Mechanical, and Electrical Engineering (TMEE) December 16-18, Changchun, China, pp698-702.

7. Wei he, Sun Wei-hua, Ma Jun-ling, Research on Multi-sensor Information Fusion Technology in Vehicle Management, 2013 International Conference on Transportation (ICTR 2013),ISBN:

978-1-60595-146-1 ,PP.165-168.

8. Visser,A.,Yakali,H.H,Van der Wees, etc.. A Hierarchical View on Modeling the Reliability of DSRC Link for ETC Applications. Intelligent Transportation

Systems, IEEE Transactions on Volume(3), 2002, pp120-129.

9.Wern-Yarng Shieh, Hsu, C.-C.J, Shen-Lung Tung, etc.. Design of Infrared Electronic-toll-collection Systems with Extended Communication Areas and Performance of Data Transmission. Intelligent Transportation Systems, IEEE Transactions on, Volume(12), 2011, pp 25 - 35.

10. Zhongmin Wen, Katayanagi, T., Arai, Y.etc.. A 5.8GHz Transmitter MMIC for Electronic Toll Collection System. Gallium Arsenide Integrated Circuit (GaAs IC) Symposium, 1998. Technical Digest 1998., 20th Annual, pp173-176. 